\title{
SOLAR-LIKE OSCILLATIONS ON $\alpha$ CENTAURI A
}

EDWARD M. POTTASCH, HARVEY R. BUTCHER and FRANS H.J. van HOESEL Kapteyn Observatory, Mensingheweg 20, 9301 KA Roden

The Netherlands.

\begin{abstract}
Detection of individual modes of oscillation near 5 minutes period is proposed, based on the comb-like signature which is similar to that found in the sun. The mean peak amplitudes $\left(\mathrm{a}_{\mathrm{p}}\right.$ ) of the strongest individual modes are $75 \leq \mathrm{a}_{\mathrm{p}} \leq 120$ $\mathrm{cm} / \mathrm{s}$, and the mean separation between alternate modes is $55.3 \pm 3.6 \mu \mathrm{Hz}$. Apparent modal lifetimes are on the order of 2 days, but we suggest this may be caused by interference of adjacent modes.
\end{abstract}

\section{INTRODUCTION}

For the past decade serious attempts (e.g. Brown and Gilliland 1990, Brown et al. 1991, Gelly et al. 1986, Noyes et al. 1984) have been made to detect solar-like p-modes on bright solar-like stars. It is expected that p-modes measured on solar-like stars will result in the determination of two parameters, $\Delta v_{0}$ and $D_{0}$, which are closely related to the sound speed integral across the solar radius and the sound speed gradient inside the nuclear burning core respectively (cf. Christensen-Dalsgaard 1984, 1988).

\section{OBSERVATIONS}

Our observations of $\alpha$ CenA were made during 6 consecutive nights, 2 - 7 April 1990, using a single channel, Fabry-Perot (F-P) based, velocity monitor with the $3.6-\mathrm{m}$ telescope of the European Southem Observatory at La Silla, Chili. The instrument makes use of a specially stabilized version of the ET-50 tunable etalon marketed by Queensgate Instruments, Ltd. (Butcher and Hicks 1986).

For the present observations, the F-P interferometer was combined with an order isolation interference filter to yield a narrow band filter, of $0.12 \AA$ FWHM tunable over \pm $50 \mathrm{~km} / \mathrm{s}$ about the FeI absorption line at $\lambda=5576 \AA$. When the filter is tuned to the line flank to provide maximum sensitivity to radial velocity, the system response was determined to be $\Delta \mathrm{I} / \mathrm{I}=4.44 \times 10^{-5}$ per $\mathrm{m} / \mathrm{s}$, where $\mathrm{I}$ is the intensity signal measured by the Si photodiode detector, and was found to be linear with velocity over the range required.

The design goal for stability of the bandpass of the interferometer of less than $1 \mathrm{~m} / \mathrm{s}$ for time scales between a few seconds and many minutes has been achieved on the one hand by incorporation of low noise etalon servo electronics, and on the other by characterizing the system response to temperature, pressure, and optical illumination variations, then stabilizing these environmental factors to the required degree. No separate, stabilized 
reference has been employed (except during test measurements to verify performance).

The velocity data time series discussed here consist of 0.2 seconds integrations taken in repeated $I_{1}-I_{2}-I_{3}-I_{4}$ sequences, where $I_{1}$ and $I_{4}$ are intensity signals with the etalon band tuned to the blue flank of the absorption line, and $I_{2}$ and $I_{3}$ are the signals on the red flank. These raw measures have been combined ('dechop') to generate a velocity sensitivity parameter $R(t)=\left(\left(I_{1}+I_{4}\right)-\left(I_{2}+I_{3}\right)\right) /\left(I_{1}+I_{2}+I_{3}+I_{4}\right)$.

\section{ANALYSIS}

The 'dechop' procedure transforms the intensity measurements into relative velocities. The resulting velocity curve shows the diumal motion of the earth's rotation, which during a typical observation period of nine hours on $\alpha$ CenA from La Silla, shows a velocity difference of about $500 \mathrm{~m} / \mathrm{s}$. For comparison we note that typical solar modes of oscillation have amplitudes in the order of $20-40 \mathrm{~cm} / \mathrm{s}$, and periods in the order of 5 minutes. The earth's rotational trend has been removed from the data by subtracting a $6^{\text {th }}$ order polynomial fit. Individual bad points (clouds, shutter closure) are replaced with linearly predicted points. Finally the data are smoothed by subtracting the convolution product with a Gaussian function from the data (full width at half height $\approx 45$ minutes). This filtering process should therefore leave any solar like oscillation signal from $\alpha \mathrm{Cen} A$ intact.

The power spectrum of the complete data set does not show a statistically significant excess power in the $3 \mathrm{mHz}$ region. Since it is expected that the power spectrum is almost periodic in frequency we calculated the power spectrum of a certain range within the power spectrum, as described by Gelly et al. (1986).

\section{RESULTS}

\subsection{Power spectra}

$\mathrm{N}$ random fluctuations, each with an rms amplitude of $A$, produce a signal of $A / \sqrt{N}$. Our stellar data show a $\mathrm{ms}\left(\sigma_{\mathrm{m}}\right)$ of $8.4 \mathrm{~m} / \mathrm{s}$ per 51.2 seconds integration time. The amplitude (a) for a continuous sine function to reach this power level in a 'white' spectrum therefore is: $\left.\sigma_{s}=\sigma_{\mathrm{m}} / \sqrt{\mathrm{N}}\right)=15 \mathrm{~cm} / \mathrm{s}$. Power spectra of two-nights sequences show slight (but not significant) enhanced power in the $3 \mathrm{mHz}$ region. The relative error of a peak $P(v)$ in the power spectrum at low frequencies ( $3 \mathrm{mHz}$ region) for peaks with a power less than 0.375 $(\mathrm{m} / \mathrm{s})^{2} / \Delta v$ (complete data series) or $1.0(\mathrm{~m} / \mathrm{s})^{2} / \Delta v$ (two-nights) is $\geq 49 \%$. Assignment of possible p-modes is discussed by Pottasch et al. (1992).

\subsection{Power spectra of power spectra}

The p-mode structure in the power spectrum consists of first order spacings $[v(n, l)-v(n$ $1, l)]$, and second order spacings $[v(n, l)-v(n-1, l+2)]$. Each peak in the Fourier domain is furthermore convolved with the Fourier transform of the window function (1/24 hours spacing). The resulting sidelobe structure can to some extent be removed by the CLEAN procedure. If the power spectrum shows a solar-like periodicity in frequency then the power spectrum of the power spectrum will reveal: 1) the distance $0.5 \Delta v_{0}$ and its higher harmonics (where $0.5 \Delta v_{0}=\langle v(n, l)-v(n-1, l)>/ 2)$ and 2) the frequency separation $\Delta v_{0}$ will be visible as a minor peak accompanied by its higher harmonics. We find that $0.5 \Delta v_{0}$ $=55.3 \pm 3.6 \mu \mathrm{Hz}$. 


\section{DISCUSSION AND CONCLUSION}

The power spectrum of the complete data set does not improve the signal to noise ratio as would be expected for modes with lifetimes in the order of 3 to 6 days, as on the sun (Libbrecht 1988). The best signal-to-noise ratio appears in two-nights data segments. When we have a closer look at the power spectra of these segments it becomes apparent that only a few major peaks occur in more than one two-nights power spectra. On the Sun (Ehgamberdiev et al. 1991) and possibly on Procyon (Brown et al. 1991) it has become evident that the occurrence of $\mathrm{p}$-modes in the power spectrum, shows a large scatter from day to day. This daily scatter in amplitude is believed mainly to be caused by interference between unresolved modes contributing to each observed peak in the power spectra. The intermittent occurrence of peaks in the successive two-nights power spectra is therefore probably caused by the combination of a short measurement interval and the particular separations of adjacent modes. Indeed the power spectrum of the complete data set shows linewidths that correspond to longer lifetimes. If interference is the cause of amplitude variations on timescales of two nights, then on days of largest amplitude the interference is likely to be constructive. Therefore we estimate the amplitudes of the individual modes consequently to be in the range: $75 \mathrm{~cm} / \mathrm{s} \leq$ amplitude $\leq 120 \mathrm{~cm} / \mathrm{s}$.

When adopting a value for $0.5 \Delta v_{0}=55.3 \pm 3.6 \mu \mathrm{Hz}(\alpha \mathrm{CenA})$ and a solar value for $\Delta v_{0}$ $=135 \mu \mathrm{Hz}$, the corresponding radius $R_{a \mathrm{CenA}}=1.17 \mathrm{R}_{\odot} \pm 0.05 \mathrm{R}_{\odot}$, since $\Delta v_{0}$ is roughly proportional to $\left(M / R^{3}\right)^{0.5}$ (where $M_{\alpha C e n A}=1.085 \mathrm{M}_{\odot}[7]$ ). Furthermore $L_{\alpha C e n A} / L_{\odot}=1.33 \pm$ 0.11 , for $\mathrm{T}_{\text {erf }}(\alpha \operatorname{CenA})=5710 \mathrm{~K}$ and $\mathrm{T}_{\text {eff }}(\mathrm{Sun})=5770 \mathrm{~K}$.

Christensen-Dalsgaard (1984) showed that first and second order frequency spacings can be used to infer the age or mass of a star $\left(\Delta v_{0}-D_{0}\right.$ diagram $)$. We find that $[v(n, 0)-v(n-$ $1,2)] / 6=D_{0} \approx 1.18 \pm 0.27$. The $\Delta v_{0}-D_{0}$ diagram yields an age for $\alpha$ CenA of $7.2 \pm 2.6$ Gy.
$(\mathrm{m} / \mathrm{s}) \sqrt{2} \Delta \mathrm{v}$
$\mathrm{m} / \mathrm{s} / \sqrt{ } \Delta \mathrm{v}$

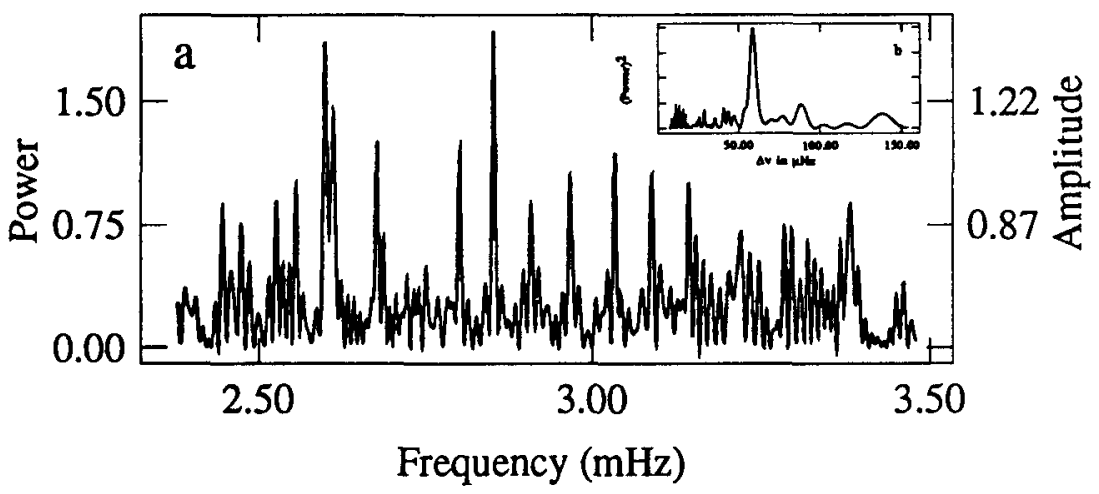

Fig.1. (a) CLEANed power spectra of two consecutive nights of observation. It is suggested that individual peaks are likely constructed by interference with neighbouring peaks. Therefore it is likely that the mode amplitudes $\left(\mathrm{a}_{\mathrm{p}}\right)$ are in the order of $75 \leq \mathrm{a}_{\mathrm{p}} \leq 120 \mathrm{~cm} / \mathrm{s}$. (b) The power spectrum of the power spectrum indicates that the p-modes are periodic in frequency space, where the spacings between the individual modes $0.5 \Delta v_{0}=55.3 \pm 3.6 \mu \mathrm{Hz}$. 


\section{REFERENCES}

Belmonte J.A., Pérez Hernández F., Roca Cortés T. 1990, A\&A $\underline{231}, 383$

Brown T.M., Gilliland R.L. 1990, ApJ $\underline{350}, 839$

Brown T.M., Gilliland R.L., Noyes R.L., Ramsay L.W. 1991, ApJ $\underline{368}$,

Butcher H.R., Hicks T.R. 1986, in Proceedings of the NATO Advanced Research Workshop on Seismology of the Sun and distant Stars, ed. D.O. Gough, p. 347

Christensen-Dalsgaard J. 1984, in Proceedings of the workshop on space research prospects in stellar activity and variability, ed. Mangeney, F. Praderie, p. 11

Christensen-Dalsgaard J. 1988, in Proceedings IAU Sym. 123: "Advances in Helio- and Asteroseismology", ed. Christensen-Dalsgaard, Frandsen, p. 295

Demarque P., Guenter D.B., van Altena W.F. 1986 ApJ $\underline{300}, 773$

Ehgamberdiev S., Khalikov S., Lazrek M., Fossat E. 1991, A\&A 253, 252

Gelly B., Grec G., Fossat E. 1986, A\&A 164,383

Libbrecht G. 1988, in Proceedings of the ESA-IAC Sym. Seismology of the sun \& sun-like stars, ed. Rolfe EJ., SP-286, p. 3

Noyes R.W., Baliunas S.L., Belserene E., Duncan D.K., Horne J., Widrow L. 1984, ApJ $\underline{285}$, L23

Pottasch E.M., Butcher H.R., van Hoesel F.H.J. 1992, A\&A (in press). 